\title{
High-throughput analysis of Yersinia pseudotuberculosis gene essentiality in optimised in vitro conditions, and implications for the speciation of Yersinia pestis
}

\author{
Samuel J. Willcocks ${ }^{1}$ (D) , Richard A. Stabler ${ }^{1}$, Helen S. Atkins ${ }^{2}$ Petra F. Oyston² and Brendan W. Wren ${ }^{1 *}$
}

\begin{abstract}
Background: Yersinia pseudotuberculosis is a zoonotic pathogen, causing mild gastrointestinal infection in humans. From this comparatively benign pathogenic species emerged the highly virulent plague bacillus, Yersinia pestis, which has experienced significant genetic divergence in a relatively short time span. Much of our knowledge of Yersinia spp. evolution stems from genomic comparison and gene expression studies. Here we apply transposon-directed insertion site sequencing (TraDIS) to describe the essential gene set of $Y$. pseudotuberculosis IP32953 in optimised in vitro growth conditions, and contrast these with the published essential genes of $Y$. pestis.

Results: The essential genes of an organism are the core genetic elements required for basic survival processes in a given growth condition, and are therefore attractive targets for antimicrobials. One such gene we identified is yptb3665, which encodes a peptide deformylase, and here we report for the first time, the sensitivity of $Y$. pseudotuberculosis to actinonin, a deformylase inhibitor. Comparison of the essential genes of Y. pseudotuberculosis with those of $Y$. pestis revealed the genes whose importance are shared by both species, as well as genes that were differentially required for growth. In particular, we find that the two species uniquely rely upon different iron acquisition and respiratory metabolic pathways under similar in vitro conditions.

Conclusions: The discovery of uniquely essential genes between the closely related Yersinia spp. represent some of the fundamental, species-defining points of divergence that arose during the evolution of $Y$. pestis from its ancestor. Furthermore, the shared essential genes represent ideal candidates for the development of novel antimicrobials against both species.
\end{abstract}

Keywords: Yersinia, TraDIS, TnSeq, Essential genes

\section{Background}

Yersinia pseudotuberculosis can colonise a wide range of animal and bird hosts, from which human infection can arise causing gastrointestinal illness. It is an important disease of young cattle in particular, and can result in chronic morbidity, or acute septicaemia, haemorrhagic diarrhoea and death [1]. The difficulty in diagnosing

\footnotetext{
* Correspondence: brendan.wren@lshtm.ac.uk

'Department of Pathogen Molecular Biology, London School of Hygiene and Tropical Medicine, Keppel Street, London WC1E 7HT, UK

Full list of author information is available at the end of the article
}

yersiniosis in both cattle and humans means that cases are likely to be considerably underreported $[2,3]$.

The study of $Y$. pseudotuberculosis has added significance because of its genetic similarity to the plague bacillus, Yersinia pestis, a recently emerged and highly virulent species [4]. The common ancestor of $Y$. pestis that is capable of causing bubonic plague is thought to have emerged in the vicinity of China some 5700 years ago $[5,6]$. That strain is itself thought to have emerged from a less virulent ancestor that diverged from $Y$. pseudotuberculosis about 54,000 years ago.

C The Author(s). 2018 Open Access This article is distributed under the terms of the Creative Commons Attribution 4.0 International License (http://creativecommons.org/licenses/by/4.0/), which permits unrestricted use, distribution, and 
The vast majority of genes present in $Y$. pseudotuberculosis share over $97 \%$ identity with the respective orthologues in $Y$. pestis [7]. While genetic conservation between the two species can be used to explain shared characteristics, genetic differences can help inform us of the evolution of virulence in $Y$. pestis. The known genetic differences between $Y$. pestis and $Y$. pseudotuberculosis to date have been described as the "foothold moments" that mark the emergence of the highly virulent species from its ancestor [8]. Such differences may include, for example, the acquisition of the plasmids pMT1 and pPCP1 by $Y$. pestis [9], as well gene inactivation by negative transcriptional regulation [10] and genome size reduction [11]. Whole genome sequencing has revealed that $Y$. pestis has approximately 100,000 fewer nucleotides than its immediate ancestor [11]. The accumulation of pseudogenes further contributes to the ongoing divergence. For example, $Y$. pseudotuberculosis IP32953 has a reported 62 pseudogenes compared to over 200 pseudogenes in strains of $Y$. pestis [7].

Gene loss may not necessarily result in a loss of function, but can sometimes facilitate the gain of a new capability. For example, $Y$. pestis has a mutation compared with $Y$. pseudotuberculosis that prevents the formation of O-antigen in its lipopolysaccharide (LPS) [12]. However, the lack of $\mathrm{O}$-antigen facilitates the activity of plasminogen activator, a plasmid-borne virulence factor found in strains of $Y$. pestis causing bubonic plague [13].

$Y$. pestis and $Y$. pseudotuberculosis occupy different niches that afford unique resources and challenges, and hence the absolute requirement of certain genes changes. For example, while $Y$. pseudotuberculosis is well adapted to survival in the soil, $Y$. pestis is less fit in this environment [14]. While both are capable of infecting mammals, the infection cycle of $Y$. pestis does not require survival in the mammalian gut, whereas it does require reaching a high cell density to cause bacteraemia [15], and can also cause pneumonic infection [16]. Finally, only $Y$. pestis is fully adapted for survival in and transmission by the flea vector $[14,17,18]$. The genetic differences between $Y$. pestis and $Y$. pseudotuberculosis that contributed to vector-borne transmission and enhanced virulence in humans are well studied, and it can be concluded that following successful adaptation of $Y$. pestis to its new niche, detrimental or superfluous genes were gradually lost [19]. Using Transposon-Directed Insertion-site Sequencing (TraDIS), also known as Transposon Sequencing (TnSeq), we are able to identify such genes in unprecedented detail.

TraDIS, or TnSeq, was first demonstrated in Salmonella Typhi [20], and has subsequently been applied to several pathogenic species including Vibrio cholerae [21], Escherichia coli [22] and Burkholderia pseudomallei [23]. Libraries are constructed from individual randomly- integrated transposon mutants that are then pooled, ranging in complexity from the small, $(<3000$ [24]), to the very large, (in excess of $1,000,000$ [23]) unique transposon mutants, saturating the genome several times over. Negative selection of transposon insertion mutants reveals their essentiality in the chosen in vitro or in vivo condition. In this study, we utilised TraDIS to establish the functional essentiality of every gene in the $Y$. pseudotuberculosis IP32953 genome under optimised in vitro growth conditions. This is in contrast to previous $Y$. pseudotuberculosis TraDIS studies that have focused on the discussion of in vivo virulence-associated genes [25].

Essential genes, particularly those that are conserved among species but with no orthologue in humans, represent potential targets for novel or re-purposed antibiotics. In the present work, we demonstrate the efficacy of actinonin, a deformylase inhibitor, against $Y$. pseudotuberculosis based on the discovery that peptide deformylase, yptb3665, is required for growth in vitro. Furthermore, we exploit the recently published $Y$. pestis essential gene data [26] and compare this to our $Y$. pseudotuberculosis TraDIS. This allowed us to contrast the differing importance of genes required for growth of either Yersinia spp. in optimised nutritional conditions. A strength of this approach is that it is not limited to only identifying the genes that are present or absent between the species, which has informed most of our knowledge of Yersinia spp. pathogen evolution to date. Also, unlike transcriptional profiling data, in which not all transcribed genes are essential, TraDIS identifies the absolute necessity of all the genes required for survival. Thus, we are able to report not only the conserved genes between $Y$. pseudotuberculosis and $Y$. pestis that are essential for growth, but also where gene essentiality has been lost, and where new genes have taken on greater significance in $Y$. pestis. These represent a record of the key sites of divergence that resulted from the emergence of $Y$. pestis from $Y$. pseudotuberculosis.

\section{Results}

The Ez-Tn5-Kan transposome complex was used to generate a library of approximately 40,000 mutants in Y. pseudotuberculosis IP32953. DNA sequencing of our 'wild-type' laboratory isolate of IP32953 revealed three non-synonymous amino-acid mutations compared with the reference sequence, these were located within: $c e l B$, a carbohydrate transporter; yptb3499, an uncharacterised gene and yptb0420, an uncharacterised gene.

Transposon coverage was mostly evenly distributed throughout the chromosome, although there was a lower density of transposon insertions between 2 and 3000 Kbps. We did not observe any apparent bias toward GC content or towards the origin of replication (Fig. 1a). In 
a
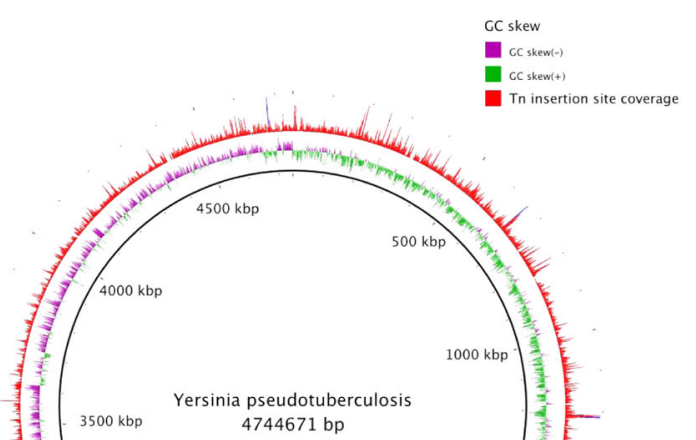

Tn insertion site coverage

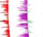

章

b

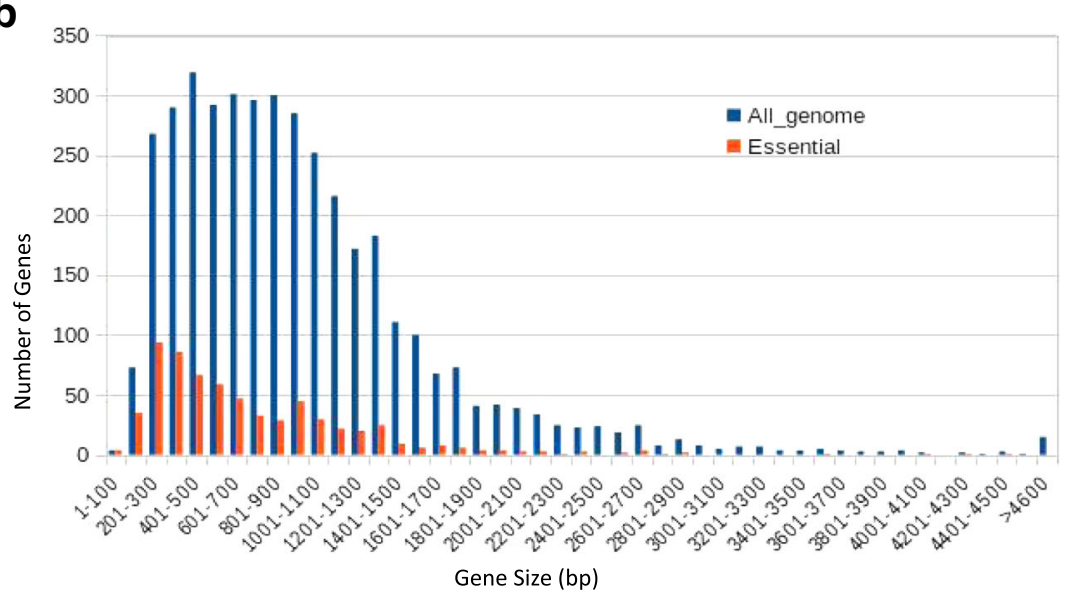

C

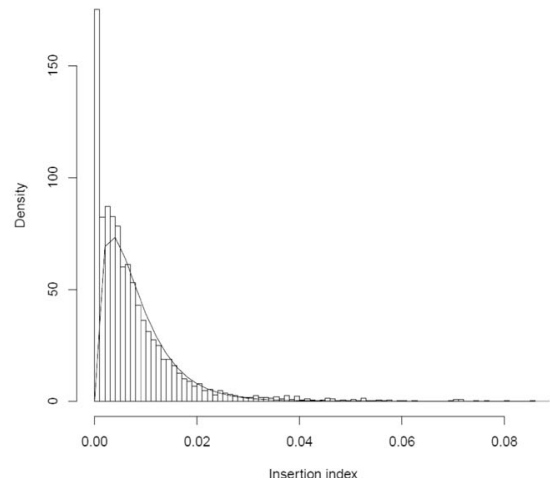

d

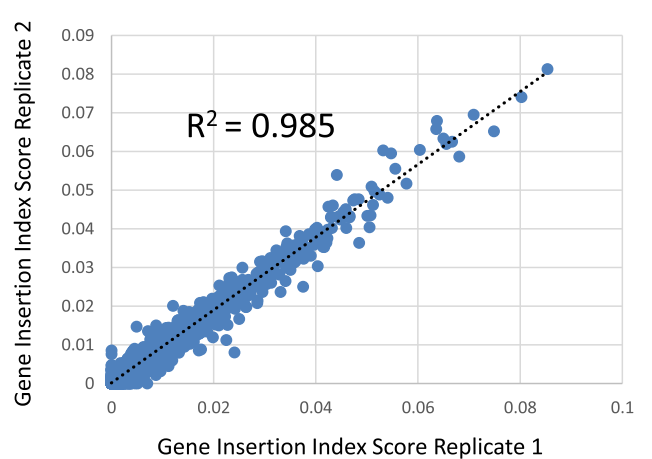

Fig. 1 (See legend on next page.) 
(See figure on previous page.)

Fig. 1 a Infographic showing the complete coverage of $\sim 40,000$ transposon insertions of Tn5 into the Y.pseudotuberculosis chromosome from the pooled TraDIS library. Transposon insertions (RED) occur evenly throughout the genome, with no obvious bias relating to high (GREEN) or low (PURPLE) GC content. Depth of reads are indicated by the height of the bars; gaps in coverage represent the location of essential genes. Inset: presence of essential genes at the muk gene cluster as visualised using Artemis software; heatmap of transposon insertions are mapped against the reference genome. Ring image generated using BRIG Blast Ring Image Generator. b Histogram showing gene-size distribution of the Y. pseudotuberculosis genome alongside gene-size distribution of identified essential genes. c Gamma-curve distribution revealing the insertion-index threshold value at which genes contain no, or very few insertions are considered to be essential. $\mathbf{d}$ Correlation coefficient between two technical replicates of TraDIS. Gene insertion index scores for every gene were compared between replicates and plotted; the coefficient score was determined using CORREL function in Microsoft Excel

general, the distribution of gene sizes identified as essential reflected the distribution of gene sizes for the genome as a whole (Fig. 1b). However, below 200 bp a higher proportion of genes were identified as essential, suggesting their small size reduced the likelihood of transposon insertion regardless of gene function. This aspect was taken into account by generating an insertion index during TraDIS data analysis as described by others [20, 27-29], which normalised insertion frequency against gene length for every gene; a low gene insertion index score is indicative of increased likelihood of essentiality. A gamma distribution curve was generated to visualise the data and calculate the threshold value at which a given gene was considered to be essential (Fig. 1c). As a quality control step, we generated a correlation coefficient between two technical replicates of the TraDIS, showing the reproducibility of the gene insertion index between replicates across the whole genome (Fig. 1d).

We generated 566,387 high-quality reads with confirmed transposon barcoding, of which 297,216 reads mapped to the reference sequence. Un-mapped reads were accounted for by phiX DNA used to facilitate sequencing; genomic DNA clusters that did not contain transposon; and reads that contained at least one mismatch with the transposon sequence. We used a custom-designed single-primer adenylation method [30] to reduce non-unique PCR amplification bias during sequencing preparation, which we conservatively limited to 10 cycles.

For assessing essentiality, we pooled the collective data from three independent sequencing reactions. The $Y$. pseudotuberculosis genome contains 4324 predicted coding sequences (CDSs), excluding non-coding RNA genes. We identified 24,480 unique genome insertion events, representing, on average, seven transposon insertions per gene, of which 3796 were disrupted by transposon insertion. An example of essential genes showing lack of transposon insertion is provided in the enlarged inset of Fig. 1a: the $m u k E / F / B$ operon, involved in chromosome condensation, segregation and cell-cycle progression.

From the resulting 534 CDSs that we identified as essential in our experimental conditions, we excluded 46 genes belonging to known phage-related genes, leaving 488 in vitro growth essential genes (Additional file 1: Table S1).
The genes we identified from in vitro growth of $Y$. pseudotuberculosis IP32953 in liquid BAB media at $28{ }^{\circ} \mathrm{C}$ are dominated by genes encoding proteins with enzymatic function, of which kinases, transferases and synthases are highly represented (summarised in Fig. 2a). The next largest group contains genes encoding proteins involved in transcription, translation and replication. Metabolic function and transporters are also a significant subset of the essential genes. Notably, several large clusters are identified such as the hem and rpl genes and those with related function. $R p l$ genes encode the $50 \mathrm{~S}$ ribosomal proteins, and hem is involved in the haeme porphyrin biosynthesis pathway.

By comparing our findings with the published $Y$. pestis in vitro essential genes [26], cultured using identical methods, we were able to identify shared and contrasting essential genes between the species (Additional file 2: Table S2). A Venn diagram showing selected shared and unique essential genes between $Y$. pseudotuberculosis and $Y$. pestis highlights some prominent trends, such differences relating to iron acquisition and oxidative phosphorylation (Fig. 2b).

We were interested to test the hypothesis that essential genes may represent targets for specific compounds that may be bactericidal. We identified yptb3665, which encodes a peptide deformylase-type enzyme, and subsequently identified that $Y$. pseudotuberculosis was sensitive to the deformylase inhibitor, actinonin (Fig. 2c).

\section{Discussion}

The essential gene set of Y. pseudotuberculosis IP32953

Previous $Y$. pseudotuberculosis transposon library screens have focused on identifying virulence genes [25, 31-33], and as such have used smaller transposon libraries that are appropriate for in vivo studies. In this study, we have screened a more complex library for essential genes required for growth in complex laboratory media.

The 485 in vitro growth essential genes we identified is comparable with other pathogens that have been assessed using similar methods; B. pseudomalle i is reported to have 505 essential genes [23]; Haemophilus influenza has 532 [34]; 636 in Pseudomonas aeruginosa [35]; 353 in Salmonella Typhimurium [36] and Clostridium difficile possesses 


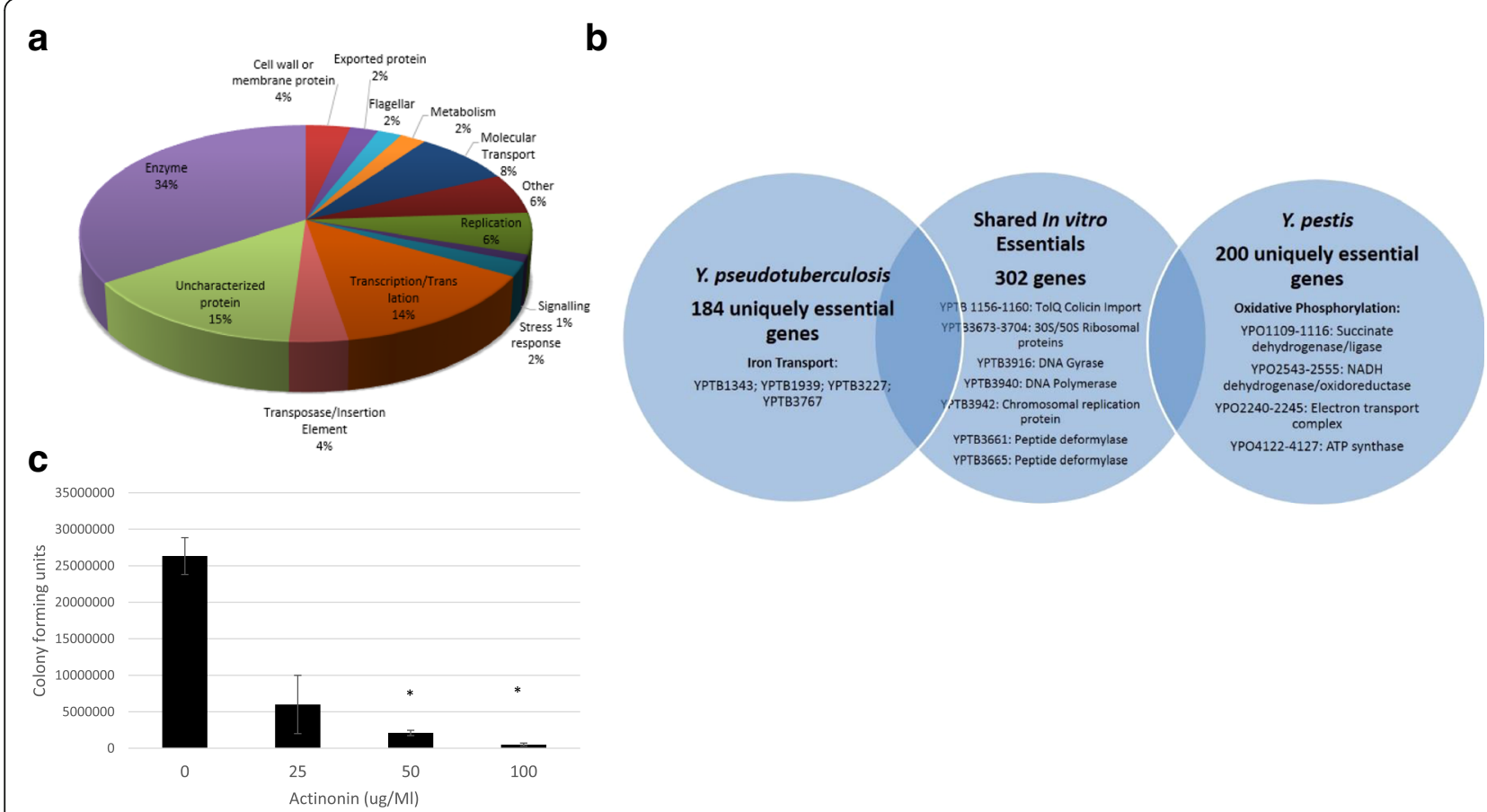

Fig. 2 a Functional Distribution of Essential Genes. The essential genes of Y. pseudotuberculosis were qualitatively assessed and grouped for their predicted function, excluding phage-related genes. $\mathbf{b}$ Venn Diagram depicting relationship between genes that were essential for in vitro growth in both Y. pseudotuberculosis and Y. pestis, and those that were found to be uniquely essential to either species. Examples of genes are provided in each condition. c Essential Genes Can Be Used to Predict Antibiotic Sensitivity. Y. pseudotuberculosis IP32953 in BAB growth media was incubated at $28^{\circ} \mathrm{C}$ with a titrated dose of actinonin, prior to enumeration of colony forming units. Student's T-test conducted versus the untreated condition $(*=0<01)$

404 essential genes [37]. A large proportion of essential genes we identified have as-yet uncharacterised function, representing a limitation in our understanding of the pathogen.

Some artefact genes were identified such as those encoding flagellar proteins, mutation of which may lower competitive fitness in liquid broth conditions without being true essential genes. Similarly, some genes have been reported to tolerate insertions at the three-prime region without complete loss of function [26]. While the risk of naming false essential genes can be reduced through adequate library complexity, biological replicates and the use of insertion-index scores, we acknowledge a margin of error inherent in any TraDIS/ Tnseq study. This stems partly from the difficulty in imposing binary bioinformatics-based thresholds of 'essentiality' on a biological system that instead operates in non-discrete degrees of fitness.

\section{TraDIS-informed identification of potential drug targets}

The identification of essential genes can contribute to the identification of novel antibacterial drug targets. Two types of essential genes have been proposed as novel targets for antimicrobial development: in vivo essential, or 'virulence' genes, and truly essential genes that are required for viability [38]. Whilst virulence genes make attractive targets for specific pathogens, any inhibitors are narrow in their selectivity and thus there is a requirement for companion diagnostics to guide clinical use. In contrast, truly essential genes are often involved in conserved processes, and as such may represent opportunities for broader spectrum therapeutics to be developed.

To minimise potential host-toxicity, bacterial essential genes that have no orthologue in mammals are attractive targets. In our TraDIS screen, we identified yptb3665, which encodes a peptide deformylase-type enzyme that appear to be essential for bacterial growth but are not found in eukaryotic cells [39]. They are the subject of a new class of inhibitor that shows promising activity and safety in vivo [40-42]. We hypothesised that since yptb3665 was found to be essential in both $Y$. pseudotuberculosis IP32953 and Y. pestis CO92 (ypo0240), then exposure to specific inhibitors should reduce viability. We were able to demonstrate that one member of this class antibiotic, actinonin, was indeed effective in inhibiting $Y$. pseudotuberculosis. Although yptb3665 itself is not widely conserved outside the genus, it is conserved among $Y$. pseudotuberculosis strains. Actinonin and related compounds have shown activity against various other deformylases 
across a wide range of bacteria, including E. coli, Staphylococcus aureus, Haemophilus influenzae and Streptococcus pneumonia [43, 44], but to our knowledge this is the first study demonstrating that a peptide deformylase is essential in a Yersinia species, and that $Y$. pseudotuberculosis it is sensitive to a peptide deformylase inhibitor. Further investigation would be required to prove that yptb3665 is the specific target of actinonin.

\section{Identification of phage and associated genes by TraDIS}

An unexpected finding in our TraDIS 'essential' dataset are the numerous genes encoding bacteriophage proteins. We do not consider it likely that genes encoding structural phage proteins such as the capsid or tail are related to bacterial fitness, although interestingly, a TnSeq study of bacteriophage similarly found that some genes encoding structural proteins resisted transposon insertion [45]. Our findings may partly be an artefact due to several of the phage genes having very short sequences, making them less likely to receive a transposon insertion. However, given the level of saturation we achieved in the library, it is still surprising that we identify so many phage genes. A number of the phage genes are responsible for the maintenance of lysogeny, interruption of which may trigger entry into the lytic cycle [46], and indeed these have been identified in previous TraDIS screens by others [35, 36, 47]. Interruption of any gene required for phage persistence or replication would also result in its absence from the overall population. Since many of these phage genes are part of a single open reading frame, transposon insertion into a non-essential gene may influence the transcription of these downstream regulatory genes.

Chromosomal DNA-protection mechanisms may also affect transposon insertion efficiency. For example, among the essential genes we identified a putative chromosomal phage toxin-antitoxin (TA) system comprised of death on curing protein (yptb1707) and prevent host-death protein, predicted to be yptb1706. Chromosomally integrated TA systems have been suggested as contributing to the genetic stability and maintenance of neighbouring genes at the locus [48-50]. We also identified predicted host restriction modification (RM) systems (yptb3879 and yptb3881) close to the phage regions. RM systems have been described as selfish genetic elements that may resist the integration of exogenous DNA by killing the host cell [51]. In addition, non-methylated transposon DNA may be susceptible to degradation by the RM at these loci [52]. Phage-encoded RM systems (potentially phage-encoded methyltransferase, yptb1797) have been experimentally demonstrated to exclude other phage and plasmid DNA [53], it is conceivable that they similarly inhibit $\operatorname{Tn} 5$ integration.

\section{Comparison with Y. pestis CO92 essential genes}

We identified a similar number of essential in vitro growth genes in $Y$. pseudotuberculosis as those found in $Y$. pestis by Yang et al. [26], and most of these genes were orthologous. These represent conserved genes whose importance has remained vital despite substantial genomic divergence by $Y$. pestis; they include genes encoding ribosomal proteins, DNA polymerase and topoisomerase. Surprisingly, both species required genes encoding colicin import proteins $(t o l A / B / R / Q)$ for in vitro replication. These periplasmic toxin transporters have been described as having a role in outer membrane stability $[54,55]$, and for this reason may be required for optimal bacterial fitness, particularly when cultured in competition with bacilli that possess functional copies of these genes.

Intriguingly, not all essential genes from $Y$. pseudotuberculosis have orthologues present in $Y$. pestis, or they exist only in the form of pseudogenes. Conversely, $Y$. pestis also possesses unique essential genes. While comparing data from two individual labs demands caution, the fact that we matched our growth conditions suggests the broad trends we reveal warrant further examination.

Since the core processes that are required for cell viability are conserved, the unique essential genes detected from our in vitro assay tended to occur in genes involved in metabolic function. Some of these species-defining differences have already been described in Yersinia spp. For example, $Y$. pestis has undergone host specialisation resulting in reduced metabolic flexibility [8] with consequences including the loss of ability to metabolise rhamnose and meliobiose, and the inability to synthesise L-isoleucine or L-methionine (reviewed by Heroven and Dersch [56]). Such changes, arising from in vivo adaptation, alter the relative importance of orthologous metabolic genes, which can be detected in vitro.

In the present study, we identified yptb0245, a putative temperature-sensitive transcriptional repressor important in cell division [57] that is not conserved in $Y$. pestis [7]. We also identified yptb2201, a predicted sodium-proton antiporter with a role in salt and $\mathrm{pH}$ stress tolerance that is absent in $Y$. pestis. This is in agreement with the findings of Pouillot et al., who show that yptb2201 is part of a cluster of genes that are required for optimal growth of $Y$. pseudotuberculosis at $28{ }^{\circ} \mathrm{C}$, but have been lost during the emergence of $Y$. pestis [58]. It is possible that the loss of yptb2201 may be compensated for by the $n h a A / B$ genes, which similarly have a role in sodium-proton anti-port and are essential for the virulence of $Y$. pestis [59].

Interestingly, the two species had unique in vitro essential genes involved in LPS modification suggesting different, but equally vital cell-wall and $\mathrm{O}$-antigen biosynthesis pathways. This reflects published phenotypic differences between the two species, such as the 
rough-type LPS produced by $Y$. pestis versus the smooth-type LPS of $Y$. pseudotuberculosis [60, 61]. Genetic shuffling of O-antigen biosynthesis genes during species divergence has similarly been reported in TraDIS comparisons between the essential genes of the closely related Salmonella serovars, Typhi and Typhimurium [36].

Both Yersinia spp. have essential genes relating to the phosphotransferase system (PTS), a translocation system responsible for the uptake of various carbohydrate substrates. However, while $Y$. pseudotuberculosis has an essential PTS gene specific to $\mathrm{N}$-acetylgalactosamine uptake (yptb3081), a glycan particularly associated with intestinal mucosal surfaces [62], $Y$. pestis has a glucosespecific PTS component (ypo2995), appropriate for a pathogen adapted to replicating in the blood, where glucose is the dominant sugar available. These differences may also be explained by the fact that glucose uptake via PTS is potentially toxic for many enterobacteriacae due to the deleterious accumulation of glucose-6-phosphate [56].

Interestingly, we detected five LysR/DeoR transcriptional regulators (yptb1518, yptb1927, yptb2301, yptb2533 and yptb3075) uniquely essential to $Y$. pseudotuberculosis, suggesting a non-redundant role in metabolism that is of diminished importance in $Y$. pestis. Similar regulators have been reported in $Y$. pestis as being conditionally upregulated in the flea, including rovM [63] and $y f b A$, which is required for biofilm formation and colonisation of the insect vector [64], but no such regulators were required for in vitro growth according to the published gene set [26].

\section{Genes for oxidative phosphorylation and iron acquisition are differentially essential in $Y$. pseudotuberculosis and $Y$. pestis under similar in vitro growth conditions}

While haeme-synthesis genes were found to be essential to both $Y$. pseudotuberculosis and $Y$. pestis in vitro, the requirement for iron-uptake mechanisms differed. We identified four essential genes encoding iron transporters: yptb3767 (feoA), yptb1939, yptb3227 and yptb1343 in Y. pseudotuberculosis that were not essential in $Y$. pestis. These genes encode proteins for the transport of both ionic ferrous iron (yptb3767 and yptb1939) and ferric iron-binding siderophores (yptb3227 and yptb1343). It is interesting that these should become apparent during in vitro culture where iron is not limited, and it suggests a degree of inflexibility in which pathways are utilised between the species. Similar differences in essentiality have been noted previously: mutation of the haeme uptake gene, $h m u S$ is not deleterious to $Y$. pestis, but mutation of its orthologue in $Y$. enterolitica is lethal [65]. Meanwhile, Barquist and colleagues used TraDIS to identify differences in gene essentiality between the mainly enteric $S$. Typhimurium and the mainly blood-colonising serovar, $S$. Typhi relating to iron acquisition [36]. Perhaps such differences represent specialisation to alternate microenvironments, but additional factors such as disruption of certain iron acquisition systems by mobile insertion elements may have enforced the evolution of diverse iron transporters [65].

The essentiality of yptb3227 and yptb1343 in strain IP32953 was unexpected, for whilst Y. pseudotuberculosis is known to encode several siderophores, they are not all present in every strain, and many are non-functional [66]. Yersiniabactin is carried by some strains on a mobile pathogenicity island, and other highly-virulent strains possess the yersiniabactin homologues, pseudochelin and yersiniachelin [66], but they are not required for replication in the gut. However, siderophore production during enteric infection in other species has been shown to be particularly important during the host inflammatory response (reviewed by Kortman et al. [67]). BLAST analysis of eleven additional strains of $Y$. pseudotuberculosis confirmed that yptb3227 and yptb1343 are chromosomally conserved, suggesting these siderophores in particular are of special importance for fitness.

Differences in iron availability and acquisition in the contrasting biological niches occupied by $Y$. pestis and $Y$. pseudotuberculosis are well documented. In vivo, $Y$. pseudotuberculosis is adapted to scavenging iron in a variety of different states through diverse, non-redundant pathways in the mammalian gut [68]. By contrast, $Y$. pestis does not require replication in the gut, and encounters mainly haeme-, lactoferrin- or transferrin-bound iron in the blood [69]. In bubonic [65] and pneumonic forms of plague, as well as in blood-plasma, ferric iron transport enabled by siderophores plays a more prominent role than ferrous iron transport [65, 70]; whilst in the flea, iron transport systems are not highly expressed [71, 72]. Y. pseudotuberculosis similarly upregulates ferric-binding siderophores when cultured in blood-plasma [73]. During aerobic growth in vitro, where ferric iron is freely available, similar to our experimental conditions, yersiniabactin is the favoured route of iron uptake by $Y$. pestis [74]. However, whilst yersiniabactin is required for virulence in peripheral tissue, it is dispensable in the bloodstream [75], as are the haeme transporters, Hmu and Has [76, 77]. $Y$. pestis may therefore have become a siderophore specialist, encoding many such transporters and providing a high degree of redundancy that is not the case for $Y$. pseudotuberculosis [78].

Besides the siderophores, ferrous-iron transporters including yptb3767 (feoA) were essential to Y. pseudotuberculosis but not to $Y$. pestis. During aerobic growth of $Y$. pestis, both ferric and ferrous iron uptake systems are generally repressed by the Fe-fur regulon; the exception being the feo system, which is repressed in the presence of iron only in microaerophilic conditions [79]. Feo may therefore be expected to be equally important to $Y$. pestis 
as to $Y$. pseudotuberculosis during iron-replete aerobic growth, but its mutation is known to be compensated for by the yfe system. While $Y$. pseudotuberculosis also possesses the $y f e$ system, both it and the feo system may be under different transcriptional regulation than in $Y$. pestis, altering the significance of these genes under similar aerobic and nutritional conditions.

The influence of oxygen saturation on iron acquisition regulation is particularly interesting since comparison of the TraDIS data also revealed the unique dependence of $Y$. pestis on genes involved in the tricarboxylic acid (TCA) cycle and oxidative phosphorylation. These included members of the suc, sdh, nuo and cyo operons, as well as genes encoding ATP synthase subunits and components of the electron transport chain $[26,33]$. This suggests that unlike $Y$. pseudotuberculosis, $Y$. pestis favours aerobic respiration under similar in vitro growth conditions. This may again reflect its adaptation to replication in the blood stream, which is nutritionally rich and highly oxygenated compared with the gut [80]. Since there is potential cross-talk in the transcriptional and metabolic pathways that are influenced by the presence of both oxygen and iron [81-86], the differing response of the two Yersinia species to available oxygen may in turn affect the regulation and importance of different iron acquisition systems.

\section{Conclusion}

By constructing a transposon library in Y. pseudotuberculosis and utilising TraDIS, we have been able to present the essential gene set for this species under defined growth conditions. As well as providing a resource for understanding its biology, we also demonstrate its utility in revealing new targets for the development or repurposing of existing antimicrobials, and report for the first time the sensitivity of $Y$. pseudotuberculosis to the deformylase inhibitor, actinonin. Comparison of our TraDIS data with those from a similar study performed with $Y$. pestis allowed us to identify fundamental differences in the importance of genes between $Y$. pestis and $Y$. pseudotuberculosis. Our results suggest that the two closely related species do not just favour, but depend upon, different iron uptake and metabolic pathways for survival, reflecting their adaptation to different microenvironments.

\section{Methods}

\section{TraDIS library preparation}

The Ez-Tn5 Kan2 transposome complex (Epicentre, UK), was electroporated into fresh electro-competent $Y$. pseudotuberculosis IP32953 (a kind gift from Prof Richard Titball, Exeter University) at $1800 \mathrm{~V}$ and recovered at $28{ }^{\circ} \mathrm{C}$ in super-optimal broth with catabolite repression (SOC) media for two hours before spreading onto Yersinia selective agar base (Oxoid, UK) infused with kanamycin at $50 \mu \mathrm{g} \mathrm{ml}^{-1}$. Resulting colonies, each representing unique transposon clones were removed from the agar and pooled in approximately 650 mutant batches in $40 \%$ glycerol until a total library size of about 40,000 mutants was achieved, and stored at $-80^{\circ} \mathrm{C}$. Transposon integration was validated by Southern blot and Linker PCR using standard methodology (data not shown).

In preparation for sequencing, the TraDIS library was thawed on ice and pooled together prior to genomic DNA extraction (ArchivePure, 5 Prime), or used to seed sterile Oxoid blood agar base no. 2 media (BAB) (ThermoScientific) for growth at $28{ }^{\circ} \mathrm{C}$ overnight for essential gene identification experiments.

Extracted genomic DNA was fragmented by sonication and size-selected by gel electrophoresis. Gel extracted DNA was assessed for size, purity and concentration by Bioanalyser $^{\text {Tw. }}$. The fragmented gDNA was end-repaired using Klenow DNA polymerase (New England Biolabs, NEB) and T4 DNA polymerase (NEB) prior to single-primer adenylation PCR of fragments containing the transposon insert [30]. This method uses a blend of A-tailing and high-fidelity enzyme to enrich transposonpositive fragments without the need for excessive PCR cycles that may introduce amplification bias. Adapters P5 and P7 were then ligated onto the A-tailed transposon-containing fragments to serve both as the template for the sequencing primer and to allow annealing to the flow cell. Finally, samples were PCR-amplified using primers specific for the P5 and P7 adapters, with a maximum of ten cycles. Prepared DNA was sequenced using Illumina MiSeq technology.

\section{Sequence analysis}

Raw sequencing data from three independent repeats in FASTQ format were analysed for the presence of the terminal ten base pair nucleotides of the transposon sequence with no mismatches, and then the transposon sequence was trimmed using a custom PERL script. Reads were curated for quality control and trimmed to 36 base pairs using Trimmomatic software. Bowtie software was used to map reads against the reference genome (European Molecular Biology Laboratory accession number: BX936398) with no mismatches allowed to establish the transposon insertion sites; non-unique mapping reads were discarded.

The TraDIS toolkit [87] determines gene essentiality based on the empirically observed bimodal distribution of insertion sites over genes when normalized for gene length using a method adapted from Langridge et al. [20] and Barquist et al. [36]. Briefly, a loess curve is fit to the distribution of insertion indices and used to fit a gamma distribution curve from which log-odds ratios are generated to determine the threshold for gene essentiality. Pseudogenes, and genes that were not conserved among multiple strains as identified by BLAST were 
excluded as false positives (data not shown). For cross-referencing against the $Y$. pestis genome, we referred to the recently published work by Yang and colleagues [26].

\section{Antibiotic sensitivity testing}

To test the activity of actinonin against $Y$. pseudotuberculosis, we incubated approximately $1 \times 10^{5}$ bacteria in 96-well plate format for $24 \mathrm{~h}$ at $28{ }^{\circ} \mathrm{C}$ in $\mathrm{BAB}$ broth with a titration of actinonin (in water solution) in triplicate prior to enumeration by serial dilution and assessment of colony forming units (CFU) growth.

\section{Additional files}

Additional file 1: Table S1. Essential Genes List Y. pseudotuberculosis IP32953 in BAB Liquid Culture $28^{\circ} \mathrm{C}$. The essential genes under defined in vitro conditions were calculated from raw sequencing data (see Methods) and listed; note that phage-related 'essential' genes are listed separately. (XLSX $32 \mathrm{~kb}$ )

Additional file 2: Table S2. Comparison of $Y$. pseudotuberculosis In vitro Essential Genes with Published Y. pestis Essential Genes. The essential genes that we identified in Y. pseudotuberculosis were cross-referenced against the published $Y$. pestis essential genes under matched conditions (Yang et al. [26]). The shared, and unique essential genes of both species are listed. (XLSX $34 \mathrm{~kb}$ )

\section{Abbreviations}

ATP: Adenosine triphosphate; BLAST: Basic local alignment search tool; CDS: Coding sequence; CFU: Colony forming units; DNA: Deoxyribonucleic acid; LPS: Lipopolysaccharide; PCR: Polymerase chain reaction; RM: Restriction modification; RNA: Ribonucleic acid; SOC: Super-optimal broth with catabolite repression; TA: Toxin-antitoxin; TCA: Tricarboxylic acid; TnSeq: Transposon sequencing; TraDIS: Transposon-directed insertion site sequencing

\section{Acknowledgements}

We thank Prof Richard Titball and Dr. Alex McCarthy for their discussions and contributions to this study.

\section{Funding}

This work was funded by the Defense Science and Technology Laboratory (DSTL), UK, who also contributed to the study design, interpretation of data and manuscript review.

\section{Availability of data and materials}

All data analysed during this study are included in this published article and its supplementary information files. Additional raw data from sequencing and quality control datasets are available from the corresponding author on reasonable request.

\section{Authors' contributions}

SW experimental design; sequencing; data analysis and main manuscript text. HA and PO design of the project; data analysis; manuscript review. RS sequencing and data analysis, manuscript review. BW Principle Investigator; design of the project; data analysis; manuscript review. All authors have read and approved this manuscript.

Ethics approval and consent to participate

Not applicable.

\section{Competing interests}

The authors declare that they have no competing interests.

\section{Publisher's Note}

Springer Nature remains neutral with regard to jurisdictional claims in published maps and institutional affiliations.

\section{Author details}

${ }^{1}$ Department of Pathogen Molecular Biology, London School of Hygiene and

Tropical Medicine, Keppel Street, London WC1E 7HT, UK. ${ }^{2}$ Microbiology, CBR

Division, DSTL Porton Down, Salisbury SP4 0JQ, UK

Received: 5 September 2017 Accepted: 18 May 2018

Published online: 31 May 2018

\section{References}

1. Slee KJ, Brightling P, Seiler RJ. Enteritis in cattle due to Yersinia pseudotuberculosis infection. Aust Vet J. 1988;65(9):271-5.

2. Amphlett A. Far East Scarlet-Like Fever: A Review of the Epidemiology, Symptomatology, and Role of Superantigenic Toxin: Yersinia pseudotuberculosisDerived Mitogen A. Open Forum Infect Dis. 2016;3(1):ofv202.

3. Galindo CL, Rosenzweig JA, Kirtley ML, Chopra AK. Pathogenesis of $Y$ enterocolitica and $Y$ pseudotuberculosis in Human Yersiniosis. J Pathog. 2011;2011:182051.

4. Achtman M, Morelli G, Zhu P, Wirth T, Diehl I, Kusecek B, et al. Microevolution and history of the plague bacillus, Yersinia pestis. Proc Natl Acad Sci U S A. 2004:101(51):17837-42.

5. Rasmussen S, Allentoft ME, Nielsen K, Orlando L, Sikora M, Sjogren KG, et al. Early divergent strains of Yersinia pestis in Eurasia 5,000 years ago. Cell. 2015;163(3):571-82.

6. Morelli G, Song Y, Mazzoni CJ, Eppinger M, Roumagnac P, Wagner DM, et al Yersinia pestis genome sequencing identifies patterns of global phylogenetic diversity. Nat Genet. 2010;42(12):1140-3.

7. Chain PS, Carniel E, Larimer FW, Lamerdin J, Stoutland PO, Regala WM, et al. Insights into the evolution of Yersinia pestis through whole-genome comparison with Yersinia pseudotuberculosis. Proc Natl Acad Sci U S A. 2004;101(38):13826-31

8. Reuter S, Connor TR, Barquist L, Walker D, Feltwell T, Harris SR, et al. Parallel independent evolution of pathogenicity within the genus Yersinia. Proc Natl Acad Sci U S A. 2014;111(18):6768-73.

9. Collyn F, Guy L, Marceau M, Simonet M, Roten CA. Describing ancient horizontal gene transfers at the nucleotide and gene levels by comparative pathogenicity island genometrics. Bioinformatics. 2006;22(9):1072-9.

10. Chauvaux S, Dillies MA, Marceau M, Rosso ML, Rousseau S, Moszer I, et al. In silico comparison of Yersinia pestis and Yersinia pseudotuberculosis transcriptomes reveals a higher expression level of crucial virulence determinants in the plague bacillus. Int J Med Microbiol. 2011;301(2):105-16.

11. Parkhill J, Wren BW, Thomson NR, Titball RW, Holden MT, Prentice MB, et al. Genome sequence of Yersinia pestis, the causative agent of plague. Nature. 2001;413(6855):523-7.

12. Skurnik M, Peippo A, Ervelä E. Characterization of the O-antigen gene clusters of Yersinia pseudotuberculosis and the cryptic O-antigen gene cluster of Yersinia pestis shows that the plague bacillus is most closely related to and has evolved from Y. pseudotuberculosis serotype O:1b. Mol Microbiol. 2000:37(2):316-30.

13. Pouillot F, Derbise A, Kukkonen M, Foulon J, Korhonen TK, Carniel E. Evaluation of O-antigen inactivation on Pla activity and virulence of Yersinia pseudotuberculosis harbouring the pPla plasmid. Microbiology. 2005;151(Pt 11):3759-68.

14. Eisen RJ, Petersen JM, Higgins CL, Wong D, Levy CE, Mead PS, et al. Persistence of Yersinia pestis in soil under natural conditions. Emerg Infect Dis. 2008;14(6):941-3.

15. Lorange EA, Race BL, Sebbane F, Hinnebusch BJ. Poor vector competence of fleas and the evolution of hypervirulence in Yersinia pestis. J Infect Dis. 2005;191(11):1907-12.

16. W. L-T. Geneva: league of nations health organization;; a treatise on pneumonic plague. 1926.

17. Buzoleva LS, Somov GP. Adaptation variability of Yersinia pseudotuberculosis during long-term persistence in soil. Bull Exp Biol Med. 2003:135(5):456-9.

18. Hinnebusch BJ, Erickson DL. Yersinia pestis biofilm in the flea vector and its role in the transmission of plague. Curr Top Microbiol Immunol. 2008:322: 229-48. 
19. Hinnebusch BJ, Chouikha I, Sun YC. Ecological opportunity, evolution, and the emergence of flea-borne plague. Infect Immun. 2016;84(7):1932-40.

20. Langridge GC, Phan MD, Turner DJ, Perkins $\Pi$, Parts L, Haase J, et al. Simultaneous assay of every Salmonella Typhi gene using one million transposon mutants. Genome Res. 2009;19(12):2308-16.

21. Chao MC, Pritchard JR, Zhang YJ, Rubin EJ, Livny J, Davis BM, et al. Highresolution definition of the Vibrio cholerae essential gene set with hidden Markov model-based analyses of transposon-insertion sequencing data. Nucleic Acids Res. 2013;41(19):9033-48.

22. Subashchandrabose S, Smith SN, Spurbeck RR, Kole MM, Mobley HL. Genome-wide detection of fitness genes in uropathogenic Escherichia coli during systemic infection. PLoS Pathog. 2013;9(12):e1003788.

23. Moule MG, Hemsley CM, Seet Q, Guerra-Assuncao JA, Lim J, Sarkar-Tyson M, et al. Genome-wide saturation mutagenesis of Burkholderia pseudomallei K96243 predicts essential genes and novel targets for antimicrobial development. MBio. 2014;5(1):e00926-13.

24. Fu Y, Waldor MK, Mekalanos JJ. Tn-Seq analysis of Vibrio cholerae intestina colonization reveals a role for T6SS-mediated antibacterial activity in the host. Cell Host Microbe. 2013;14(6):652-63.

25. Crimmins GT, Mohammadi S, Green ER, Bergman MA, Isberg RR, Mecsas J. Identification of MrtAB, an $A B C$ transporter specifically required for Yersinia pseudotuberculosis to colonize the mesenteric lymph nodes. PLoS Pathog. 2012;8(8):e1002828.

26. Yang ZR, Bullifent HL, Moore K, Paszkiewicz K, Saint RJ, Southern SJ, et al. A noise trimming and positional significance of transposon insertion system to identify essential genes in Yersinia pestis. Sci Rep. 2017;7:41923.

27. Christen B, Abeliuk E, Collier JM, Kalogeraki VS, Passarelli B, Coller JA, et al. The essential genome of a bacterium. Mol Syst Biol. 2011;7:528.

28. Phan MD, Peters KM, Sarkar S, Lukowski SW, Allsopp LP, Gomes Moriel D, et al. The serum resistome of a globally disseminated multidrug resistant uropathogenic Escherichia coli clone. PLoS Genet. 2013;9(10):e1003834.

29. Curtis PD, Brun YV. Identification of essential alphaproteobacterial genes reveals operational variability in conserved developmental and cell cycle systems. Mol Microbiol. 2014;93(4):713-35.

30. Willcocks SS, R; Wren, B. More Signal, Less Noise: Single-primer Adenylation Method (SAM) for the Efficient Preparation of DNA for Transposon Sequencing (TnSeq). biorxiv. [Preprint]. In press 2014.

31. Pradel E, Lemaitre N, Merchez M, Ricard I, Reboul A, Dewitte A, et al. New insights into how Yersinia pestis adapts to its mammalian host during bubonic plague. PLoS Pathog. 2014;10(3):e1004029.

32. Ponnusamy D, Fitts EC, Sha J, Erova TE, Kozlova EV, Kirtley ML, et al. Highthroughput, signature-tagged mutagenic approach to identify novel virulence factors of Yersinia pestis CO92 in a mouse model of infection. Infect Immun. 2015;83(5):2065-81.

33. Palace SG, Proulx MK, Lu S, Baker RE, Goguen JD. Genome-wide mutant fitness profiling identifies nutritional requirements for optimal growth of Yersinia pestis in deep tissue. MBio. 2014;5(4)

34. Mobegi FM, van Hijum SA, Burghout P, Bootsma HJ, de Vries SP, van der Gaast-de Jongh CE, et al. From microbial gene essentiality to novel antimicrobial drug targets. BMC Genomics. 2014;15:958

35. Skurnik D, Roux D, Aschard H, Cattoir V, Yoder-Himes D, Lory S, et al. A comprehensive analysis of in vitro and in vivo genetic fitness of Pseudomonas aeruginosa using high-throughput sequencing of transposon libraries. PLoS Pathog. 2013;9(9):e1003582.

36. Barquist L, Langridge GC, Turner DJ, Phan MD, Turner AK, Bateman A, et al. A comparison of dense transposon insertion libraries in the Salmonella serovars Typhi and typhimurium. Nucleic Acids Res. 2013;41(8):4549-64.

37. Dembek M, Barquist L, Boinett CJ, Cain AK, Mayho M, Lawley TD, et al. Highthroughput analysis of gene essentiality and sporulation in Clostridium difficile. MBio. 2015;6(2):e02383.

38. Klein BA, Duncan MJ, Hu LT. Defining essential genes and identifying virulence factors of Porphyromonas gingivalis by massively parallel sequencing of transposon libraries (Tn-seq). Methods Mol Biol. 2015;1279:25-43.

39. Becker A, Schlichting I, Kabsch W, Groche D, Schultz S, Wagner AF. Iron center, substrate recognition and mechanism of peptide deformylase. Nat Struct Biol. 1998;5(12):1053-8.

40. Jain $R$, Chen D, White RJ, Patel DV, Yuan Z. Bacterial peptide deformylase inhibitors: a new class of antibacterial agents. Curr Med Chem. 2005;12(14):1607-21.

41. O'Dwyer K, Hackel M, Hightower S, Hoban D, Bouchillon S, Qin D, et al. Comparative analysis of the antibacterial activity of a novel peptide deformylase inhibitor, GSK1322322. Antimicrob Agents Chemother. 2013;57(5):2333-42.
42. Guay DR. Drug forecast - the peptide deformylase inhibitors as antibacterial agents. Ther Clin Risk Manag. 2007;3(4):513-25.

43. Clements JM, Beckett RP, Brown A, Catlin G, Lobell M, Palan S, et al. Antibiotic activity and characterization of BB-3497, a novel peptide deformylase inhibitor. Antimicrob Agents Chemother. 2001;45(2):563-70.

44. Chen DZ, Patel DV, Hackbarth CJ, Wang W, Dreyer G, Young DC, et al. Actinonin, a naturally occurring antibacterial agent, is a potent deformylase inhibitor. Biochemistry. 2000;39(6):1256-62.

45. Krupovic M, Vilen H, Bamford JK, Kivela HM, Aalto JM, Savilahti H, et al. Genome characterization of lipid-containing marine bacteriophage PM2 by transposon insertion mutagenesis. J Virol. 2006;80(18):9270-8.

46. Kim M, Ryu S. Antirepression system associated with the life cycle switch in the temperate podoviridae phage SPC32H. J Virol. 2013;87(21):11775-86.

47. Le Breton Y, Belew AT, Valdes KM, Islam E, Curry P, Tettelin H, et al. Essential genes in the Core genome of the human pathogen Streptococcus pyogenes. Sci Rep. 2015;5:9838.

48. Van Melderen L, Saavedra De Bast M. Bacterial toxin-antitoxin systems: more than selfish entities? PLoS Genet. 2009;5(3):e1000437.

49. Christensen-Dalsgaard M, Gerdes K. Two higBA loci in the Vibrio cholerae superintegron encode mRNA cleaving enzymes and can stabilize plasmids. Mol Microbiol. 2006;62(2):397-411.

50. Rowe-Magnus DA, Guerout AM, Biskri L, Bouige P, Mazel D. Comparative analysis of superintegrons: engineering extensive genetic diversity in the Vibrionaceae. Genome Res. 2003;13(3):428-42.

51. Kobayashi I. Behavior of restriction-modification systems as selfish mobile elements and their impact on genome evolution. Nucleic Acids Res. 2001; 29(18):3742-56.

52. Katsube T, Matsumoto S, Takatsuka M, Okuyama M, Ozeki Y, Naito M, et al. Control of cell wall assembly by a histone-like protein in mycobacteria. J Bacteriol. 2007:189(22):8241-9.

53. van der Mee-Marquet N, Corvaglia AR, Valentin AS, Hernandez D, Bertrand X, Girard M, et al. Analysis of prophages harbored by the human-adapted subpopulation of Staphylococcus aureus CC398. Infect Genet Evol. 2013;18: 299-308.

54. Ridley $\mathrm{H}$, Lakey $\mathrm{JH}$. Antibacterial toxin colicin $\mathrm{N}$ and phage protein G3p compete with TolB for a binding site on TolA. Microbiology. 2015;161(Pt 3):503-15.

55. Li C, Zhang Y, Vankemmelbeke M, Hecht O, Aleanizy FS, Macdonald C, et al. Structural evidence that colicin a protein binds to a novel binding site of TolA protein in Escherichia coli periplasm. J Biol Chem. 2012;287(23):19048-57.

56. Heroven AK, Dersch P. Coregulation of host-adapted metabolism and virulence by pathogenic yersiniae. Front Cell Infect Microbiol. 2014;4:146.

57. Bejar S, Bouche JP. A new dispensable genetic locus of the terminus region involved in control of cell division in Escherichia coli. Mol Gen Genet. 1985; 201(2):146-50

58. Pouillot F, Fayolle C, Carniel E. Characterization of chromosomal regions conserved in Yersinia pseudotuberculosis and lost by Yersinia pestis. Infect Immun. 2008;76(10):4592-9.

59. Minato Y, Ghosh A, Faulkner WJ, Lind EJ, Schesser Bartra S, Plano GV, et al. $\mathrm{Na}+/ \mathrm{H}+$ antiport is essential for Yersinia pestis virulence. Infect Immun. 2013:81(9):3163-72.

60. Kukkonen M, Suomalainen M, Kyllonen P, Lahteenmaki K, Lang H, Virkola R, et al. Lack of $\mathrm{O}$-antigen is essential for plasminogen activation by Yersinia pestis and Salmonella enterica. Mol Microbiol. 2004;51(1):215-25.

61. Oyston PC, Prior JL, Kiljunen S, Skurnik M, Hill J, Titball RW. Expression of heterologous O-antigen in Yersinia pestis KIM does not affect virulence by the intravenous route. J Med Microbiol. 2003;52(Pt 4):289-94.

62. Bergstrom KS, Xia L. Mucin-type O-glycans and their roles in intestinal homeostasis. Glycobiology. 2013;23(9):1026-37.

63. Vadyvaloo V, Hinz AK. A LysR-type transcriptional regulator, RovM, senses nutritional cues suggesting that it is involved in metabolic adaptation of Yersinia pestis to the flea gut. PLoS One. 2015;10(9):e0137508.

64. Tam C, Demke O, Hermanas T, Mitchell A, Hendrickx AP, Schneewind O. YfbA, a Yersinia pestis regulator required for colonization and biofilm formation in the gut of cat fleas. J Bacteriol. 2014;196(6):1165-73.

65. Perry RD, Bobrov AG, Fetherston JD. The role of transition metal transporters for iron, zinc, manganese, and copper in the pathogenesis of Yersinia pestis. Metallomics. 2015;7(6):965-78.

66. Rakin A, Schneider L, Podladchikova O. Hunger for iron: the alternative siderophore iron scavenging systems in highly virulent Yersinia. Front Cell Infect Microbiol. 2012;2:151. 
67. Kortman GA, Raffatellu M, Swinkels DW, Tjalsma H. Nutritional iron turned inside out: intestinal stress from a gut microbial perspective. FEMS Microbiol Rev. 2014;38(6):1202-34.

68. Payne SM, Mey AR, Wyckoff EE. Vibrio Iron transport: evolutionary adaptation to life in multiple environments. Microbiol Mol Biol Rev. 2016; 80(1):69-90.

69. Parrow NL, Fleming RE, Minnick MF. Sequestration and scavenging of iron in infection. Infect Immun. 2013;81(10):3503-14.

70. Sebbane F, Lemaitre N, Sturdevant DE, Rebeil R, Virtaneva K, Porcella SF, et al. Adaptive response of Yersinia pestis to extracellular effectors of innate immunity during bubonic plague. Proc Natl Acad Sci U S A. 2006;103(31): 11766-71.

71. Hinnebusch BJ. Biofilm-dependent and biofilm-independent mechanisms of transmission of Yersinia pestis by fleas. Adv Exp Med Biol. 2012;954:237-43.

72. Vadyvaloo V, Jarrett C, Sturdevant DE, Sebbane F, Hinnebusch BJ. Transit through the flea vector induces a pretransmission innate immunity resistance phenotype in Yersinia pestis. PLoS Pathog. 2010;6(2):e1000783.

73. Rosso ML, Chauvaux S, Dessein R, Laurans C, Frangeul L, Lacroix C, et al. Growth of Yersinia pseudotuberculosis in human plasma: impacts on virulence and metabolic gene expression. BMC Microbiol. 2008;8:211.

74. Fetherston JD, Mier I Jr, Truszczynska H, Perry RD. The Yfe and Feo transporters are involved in microaerobic growth and virulence of Yersinia pestis in bubonic plague. Infect Immun. 2012;80(11):3880-91.

75. Kirillina O, Bobrov AG, Fetherston JD, Perry RD. Hierarchy of iron uptake systems: Yfu and Yiu are functional in Yersinia pestis. Infect Immun. 2006; 74(11):6171-8.

76. Rossi MS, Fetherston JD, Letoffe S, Carniel E, Perry RD, Ghigo JM Identification and characterization of the hemophore-dependent heme acquisition system of Yersinia pestis. Infect Immun. 2001;69(11):6707-17.

77. Forman S, Paulley JT, Fetherston JD, Cheng YQ, Perry RD. Yersinia ironomics: comparison of iron transporters among Yersinia pestis biotypes and its nearest neighbor, Yersinia pseudotuberculosis. Biometals. 2010;23(2):275-94.

78. Chauvaux S, Rosso ML, Frangeul L, Lacroix C, Labarre L, Schiavo A, et al. Transcriptome analysis of Yersinia pestis in human plasma: an approach for discovering bacterial genes involved in septicaemic plaque. Microbiology. 2007;153(Pt 9):3112-24.

79. O'Connor L, Fetherston JD, Perry RD. The feoABC locus of Yersinia pestis likely has two promoters causing unique Iron regulation. Front Cell Infect Microbiol. 2017;7:331.

80. Motin VL, Georgescu AM, Fitch JP, Gu PP, Nelson DO, Mabery SL, et al. Temporal global changes in gene expression during temperature transition in Yersinia pestis. J Bacteriol. 2004;186(18):6298-305.

81. Schwiesow L, Mettert E, Wei Y, Miller H, Herrera G, Balderas D, et al. Control of hmu Heme uptake genes in Yersinia pseudotuberculosis in response to Iron sources. Front Cell Infect Microbiol. 2018;8(47)

82. Giel JL, Rodionov D, Liu M, Blattner FR, Kiley PJ. IscR-dependent gene expression links iron-Sulphur cluster assembly to the control of O2regulated genes in Escherichia coli. Mol Microbiol. 2006;60(4):1058-75.

83. Wu Y, Outten FW. IscR controls iron-dependent biofilm formation in Escherichia coli by regulating type I fimbria expression. J Bacteriol. 2009; 191(4):1248-57.

84. Yeo WS, Lee JH, Lee KC, Roe JH. IscR acts as an activator in response to oxidative stress for the suf operon encoding Fe-S assembly proteins. Mol Microbiol. 2006;61(1):206-18.

85. Yingping F, Lemeille S, Talla E, Janicki A, Denis Y, Zhang CC, et al. Unravelling the cross-talk between iron starvation and oxidative stress responses highlights the key role of PerR (alr0957) in peroxide signalling in the cyanobacterium Nostoc PCC 7120. Environ Microbiol Rep. 2014;6(5): 468-75

86. Ledala N, Zhang B, Seravalli J, Powers R, Somerville GA. Influence of iron and aeration on Staphylococcus aureus growth, metabolism, and transcription. J Bacteriol. 2014;196(12):2178-89.

87. Barquist L, Mayho M, Cummins C, Cain AK, Boinett CJ, Page AJ, et al. The TraDIS toolkit: sequencing and analysis for dense transposon mutant libraries. Bioinformatics. 2016:32(7):1109-11.

\section{Ready to submit your research? Choose BMC and benefit from:}

- fast, convenient online submission

- thorough peer review by experienced researchers in your field

- rapid publication on acceptance

- support for research data, including large and complex data types

- gold Open Access which fosters wider collaboration and increased citations

- maximum visibility for your research: over $100 \mathrm{M}$ website views per year

At BMC, research is always in progress.

Learn more biomedcentral.com/submissions 